\title{
Effect of Smear Clear and Some Other Commonly Used Irrigants on dislodgement resistance of Mineral Trioxide Aggregate to Root Dentin
}

\author{
Mona Sadegh ${ }^{1}$, Hasti Sohrabi ${ }^{2}$, Mohamadjavad Kharazifard ${ }^{3}$, Farzaneh Afkhami ${ }^{1}$ \\ ${ }^{1}$ DDS, MSc, Department of Endodontics, Tehran University of Medical Sciences, International Campus, Tehran, Iran \\ ${ }^{2}$ DDS, Tehran University of Medical Sciences, International Campus, Tehran, Iran \\ ${ }^{3}$ DDS, PhD, Department of Epidemiology, Tehran University of Medical Sciences, Tehran, Iran
}

Correspondence:

Department of Endodontics

School of Dentistry

Tehran University of Medical Sciences

International Campus, Tehran, Iran

School of Dentistry, Mahan St.

Khaniabadno Avu., Tehran, Iran

f-afkhami@tums.ac.ir

\begin{abstract}
Sadegh M, Sohrabi H, Kharazifard M, Afkhami F. Effect of Smear Clear and Some Other Commonly Used Irrigants on dislodgement resistance of Mineral Trioxide Aggregate to Root Dentin. J Clin Exp Dent. 2017;9(5):e617-21.

http://www.medicinaoral.com/odo/volumenes/v9i5/jcedv9i5p617.pdf
\end{abstract}

Received: 06/08/2016

Accepted: $14 / 10 / 2016$

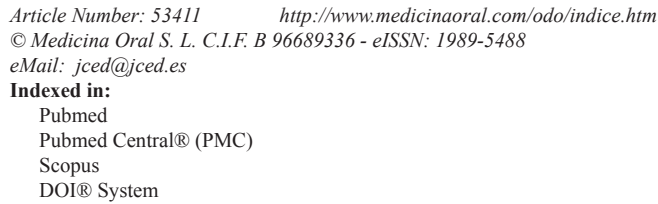

\begin{abstract}
Background: This study aimed to assess the push-out bond strength of mineral trioxide aggregate (MTA) to root canal dentin after irrigation with Smear Clear in comparison with $2.5 \%$ sodium hypochlorite $(\mathrm{NaOCl}), 2 \%$ chlorhexidine (CHX) and saline as commonly used root canal irrigants.

Material and Methods: The coronal and mid-root areas of maxillary anterior teeth were horizontally sectioned into one-millimeter thick slices. The root canal lumen of dentinal slices was dilated using a diamond bur with $1.3 \mathrm{~mm}$ diameter. After the application of MTA, the samples were incubated in $100 \%$ humidity for 10 minutes and were then randomly divided into four groups $(\mathrm{n}=20)$ and immersed in Smear Clear, $2.5 \% \mathrm{NaOCl}, 2 \% \mathrm{CHX}$ and saline for 30 minutes. No irrigant was used for the control group $(n=20)$. A wet cotton pellet was placed on the samples and after 48 hours of incubation, push-out bond strength was measured using a universal testing machine. The samples were evaluated under a stereomicroscope to determine the mode of failure. One-way ANOVA was used to assess statistical differences among the groups.

Results: The control group showed the highest bond strength with significant differences with other groups $(P<0.05)$. Among the experimental groups, the saline group had no significant difference with $\mathrm{CHX}(P=0.09)$ but it had significant differences with Smear Clear and $\mathrm{NaOCl}$ groups $(P<0.05)$. No significant difference in bond strength to MTA was noted after irrigation with Smear Clear, $\mathrm{CHX}$ and $\mathrm{NaOCl}(P>0.05)$. Other pairwise comparisons showed no significant difference $(P>0.05)$.

Conclusions: Irrigation with Smear Clear, $\mathrm{CHX}$ and $\mathrm{NaOCl}$ did not cause a significant change in bond strength of MTA to dentin.
\end{abstract}

Key words: Root Canal Irrigants, push-out, Mineral Trioxide Aggregate, dentin. 


\section{Introduction}

Mineral trioxide aggregate is an ideal calcium silicatebased restorative material composed of hydrophilic particles, hydrated calcium silicate gel and calcium hydroxide $(1,2)$. It has optimal sealing ability, biocompatibility and insolubility, excellent adhesion to dentin and radiopacity (2). Moreover, it induces the regeneration of root cementum and dentin (3). It is also used for repair of root perforations during endodontic treatment (4). Depending on the time of occurrence of root perforation in terms of the endodontic treatment phase, it must be repaired prior to finalizing the endodontic treatment in order to improve prognosis $(5,6)$. The most important shortcoming of MTA is its long setting time (4). Thus, its bond to dentin may be compromised by root canal irrigation with irrigating solutions (1).

Recently, a new formulation of ethylenediaminetetraacetic acid (EDTA) was introduced to the market with the brand name "Smear Clear" (SybronEndo, Orange, CA), which contains $17 \%$ EDTA, Cetrimide and a specific surfactant. It has higher wettability than the conventional EDTA and the manufacturer claims that it has higher cleaning efficacy than EDTA as well (7). Dunavant et al. (8) showed that Smear Clear had significant antibacterial activity against Enterococcus faecalis and had greater efficacy for elimination of biofilm than $2 \%$ CHX. Jantarat et al. (7) demonstrated that the opening of dentinal tubules was greater in the Smear Clear group; this indicated more efficient penetration of Smear Clear compared to other irrigants into dentinal tubules.

Çelik et al. (2) reported that irrigants did not compromise the push-out bond strength of calcium silicate cements to root dentin. Calcium silicate cements are currently used in endodontic procedures due to having optimal properties. However, Yan et al. (9) stated that the push-out bond strength of materials used for root perforation repair such as MTA depended on the solution in which they were immersed. Loxely et al. (10) reported that the bond strength of MTA significantly decreased following treatment with sodium perborate + saline or sodium perborate + superoxol. Bond strength of restorative materials to dentinal walls is an important factor in assessment of their sealing ability under functional masticatory forces and biomechanical loads as well as the treatment success (2). The push-out bond strength test is commonly performed for assessment of the push-out bond strength of root canal filling materials (4).

According to Collares et al., (11) several factors can affect the results of push-out bond strength testing such as the storage time of samples, load velocity of the testing machine, use of human or bovine dentin and the part of tooth to be tested. Smear layer removal was the only factor that did not affect the push-out bond strength in the study by Collares et al. (11). Pane et al. (12) concluded that the push-out test might be suitable for ranking of root canal filling materials in terms of their efficacy.

This study sought to assess the effect of Smear Clear and some other commonly used irrigants on push-out bond strength of MTA to dentin in vitro.

\section{Material and Methods}

Forty-eight extracted human single-rooted teeth were used in this study. The crowns were cut below the cementoenamel junction. The roots were mounted in a cylindrical metal mold measuring $35 \times 25 \times 10 \mathrm{~mm}$ containing auto-polymerizing acrylic resin (ACROPARS, Marlic Medical industry, Iran) and the resin was allowed to set. After polymerization, the mounted roots were separated from the mold and stored in saline.

The coronal and mid-root dentin was cut into one-millimeter thick sections by a low-speed CNC (Nemofanavaranepars, Tehran, Iran) saw under water irrigation. Next, the lumen of dentinal discs was standardized using a round diamond bur with $1.3 \mathrm{~mm}$ diameter.

A mixture of MTA (Angelus, Londrina, PR, Brasil) was prepared according to the manufacturer's instructions. It was gradually applied to the root canal lumen of dentinal slices by a carrier (D\&P, Forgeman, Pakistan) and condensed by an endodontic plugger (D\&P, Forgeman, Pakistan). Moist gel-foam (Clinisponge, yucelmedical, Turkey) was placed beneath the dentinal slices to simulate periapical tissue conditions. The excess material on the surface of slices was removed by a scalpel. All samples were evaluated under a stereomicroscope at $\times 40$ magnification (Olympus, SZ51, Taiwan). Possible cracks, defects or gaps between the material and dentinal wall were fixed. The samples were placed inside plastic bags along with moist gauze and placed in an incubator at $37^{\circ} \mathrm{C}$ and $100 \%$ moisture for 10 minutes for primary setting. The samples were then randomly divided into four groups $(\mathrm{n}=20)$ and immersed in $2.5 \% \mathrm{NaOCl}$ (Chloraxid, Cerkamed, Polska), 2\% CHX (FGM, Dentscare LTDA, Brazil), saline (Shahid Ghazi Pharmaceutical Co., Tehran, Iran) or Smear Clear (SybronEndo, Orange, CA, USA) for 30 minutes. Next, the samples were rinsed with copious distilled water and incubated at $37^{\circ} \mathrm{C}$ and $100 \%$ moisture for 48 hours. In the control group, wet cotton pellets were placed on the MTA for 48 hours in order for the MTA to set $(n=20)$.

-The push-out bond strength testing:

The push-out bond strength was measured by a universal testing machine (STM-20, Santam machine, Germany). The samples were placed on a metal slab with a central hole to allow free movement of probe. Compressive load was applied vertically at a crosshead speed of $1 \mathrm{~mm} / \mathrm{min}$. The probe had approximately $0.2 \mathrm{~mm}$ distance from the dentinal wall margin in order to be in contact with the material only. Maximum load at failure was recorded in Newton (N). The push-out bond strength was then calculated in Megapascals (MPa) using the formula N/2лrh, 
where $\mathrm{N}$ is the maximum load at failure and $\pi \mathrm{rh}$ is the bonding surface area. Mode of failure was determined under a stereomicroscope at $\times 40$ magnification (Olympus, SZ51, Taiwan) and divided into three groups of adhesive (between the material and dentinal wall), cohesive within the material or dentin and mixed (a combination of adhesive and cohesive) (Fig. 1).

The data were analyzed using SPSS 22. One-way ANOVA and post-hoc test were used to compare the bond strength among the groups. Level of significance was set at $P=0.05$.

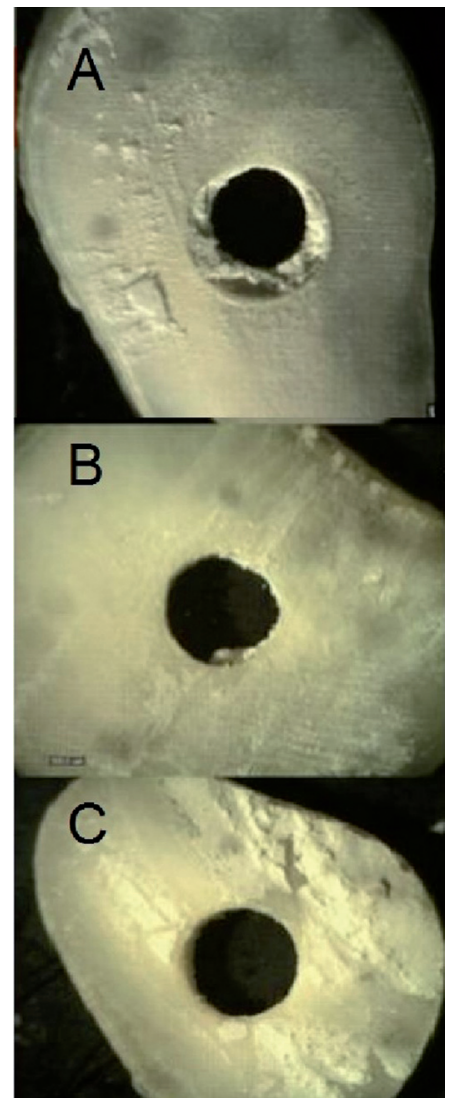

Fig. 1. Microscopic micrographs of samples at $\times 10$ magnification and mode of failure; A) Cohesive failure within MTA; B) Mixed failure, note the MTA residues in the root canal; C) Adhesive failure.

\section{Results}

Table 1 shows the mean and standard deviation of pushout bond strength in the groups. One-way ANOVA showed a significant difference in bond strength of the groups. The control group had the highest bond strength and showed statistically significant differences in this respect with Smear Clear $(P=0.012)$, CHX $(P=0.032)$ and $\mathrm{NaOCl}(P=0.006)$ groups. Also, significant differences were noted in bond strength between saline and
Smear Clear $(P=0.007)$ and saline and sodium hypochlorite $(P=0.001)$ groups. Other pairwise comparisons did not show statistically significant differences $(P>0.05)$. The mode of failure of samples are presented in table 2.

Table 1. The mean push-out bond strength and standard deviation (SD) of the samples at 48 hours (MPa).

\begin{tabular}{|l|c|c|}
\hline Group & Number & Mean \pm SD (MPa) \\
\hline Smear Clear & 20 & $0.77 \pm 0.49^{\mathrm{a}}$ \\
\hline $2.5 \% \mathrm{NaOCl}$ & 20 & $0.61 \pm 0.36^{\mathrm{a}}$ \\
\hline $2 \% \mathrm{CHX}$ & 20 & $0.93 \pm 0.98^{\mathrm{ab}}$ \\
\hline Normal Saline & 20 & $1.83 \pm 1.12^{\mathrm{bc}}$ \\
\hline Control & 20 & $2.75 \pm 2.3^{\mathrm{c}}$ \\
\hline
\end{tabular}

Different superscripted letters show statistically significant differences $(P<0.05)$.

Table 2. Mode of failure of the samples (\%).

\begin{tabular}{|l|c|c|c|}
\hline Groups & \multicolumn{3}{|c|}{ Failure mode \% } \\
\hline & Adhesive & Mixed & Cohesive \\
\hline Smear Clear & 5 & 95 & 0 \\
\hline $2.5 \% \mathrm{NaOCl}$ & 5 & 90 & 5 \\
\hline $2 \% \mathrm{CHX}$ & 5 & 85 & 10 \\
\hline $\begin{array}{l}\text { Normal } \\
\text { Saline }\end{array}$ & 0 & 90 & 10 \\
\hline Control & 5 & 85 & 10 \\
\hline
\end{tabular}

\section{Discussion}

The gray MTA cement was first developed in Loma Linda University; however, its major drawback was causing tooth discoloration due to the presence of iron compounds in its formulation. By elimination of iron compounds, white MTA was developed (13) which was used in the present study.

Sodium hypochlorite solution can non-specifically dissolve collagenous and non-collagenous proteins and adversely affect the physical properties of dentin (14). It is the most commonly recognized antimicrobial irrigant in root canal therapy (15). Yan et al. (9) showed that bond strength of dentin to MTA decreased following the use of $5.25 \% \mathrm{NaOCl}$; however, the difference with the control group was not significant in this respect. In our study, the push-out bond strength of MTA to dentin was the lowest in $2.5 \% \mathrm{NaOCl}$ group and it had a significant difference with the saline (control) group. This difference in the results of the two studies may be attributed to the different immersion periods of samples. In the study by Yan et al., the samples were immersed for two hours in saline in the control group while we immersed the samples for 30 
minutes in saline and incubated them for 48 hours. Thus, the time allowed for the setting of MTA in contact with saline in our study was longer than that in the study by Yan et al., and this resulted in higher bond strength of MTA cement. In the study by Hong et al., (1) non-accelerated MTA in contact with $2.5 \% \mathrm{NaOCl}$ had lower push-out bond strength value than the control group, which was somehow in line with our findings. Nagas et al. (16) showed that MTA treated with $5.25 \% \mathrm{NaOCl}$ had lower push-out bond strength than the control group. Another study (17) showed that samples treated with $\mathrm{NaOCl}$ had the lowest push-out bond strength value.

Chlorhexidine root canal irrigant has wide-spectrum antimicrobial activity against Gram-positive and Gramnegative bacteria. It has favorable substantivity and low cytotoxicity and thus, it is increasingly used in endodontics (18). Cecchin et al. (19) evaluated the effect of $\mathrm{CHX}$ and ethanol on the durability of the adhesion of fiber posts relined with composite resin to dentinal walls and showed that the group treated with CHX or ethanol showed equal bond strength along the root length after one day of immersion in water. They concluded that CHX and ethanol had no effect on the bond strength of fiber posts relined with composite resin to dentin. This finding was in contrast to our results, which may be due to the differences in the methodology of studies and the tested materials. Hong et al. (1) showed that CHX adversely affected the physical properties and hydration behavior of MTA. These findings were in agreement with our results since we showed that $2 \%$ CHX significantly decreased the push-out bond strength of MTA to dentin. Several studies demonstrated that bond strength of MTA significantly decreased when exposed to CHX $(6,20,21)$.

Guneser et al. (21) indicated that MTA treated with saline had higher bond strength than the control MTA group; this finding was in contrast to our results since in our study, the push-out bond strength of the control group was higher than that of MTA treated with saline, but this difference did not reach statistical significance $(P>0.05)$. Reyes-Carmona et al. (22) evaluated the biomineralization ability of MTA in presence of phosphate buffered saline (PBS) to increase its push-out bond strength to dentin and concluded that samples immersed in PBS had significantly higher bond strength at three days compared to those exposed to wet cotton pellets. Saline positively affects the size of MTA crystals (21) and completes the process of MTA hydration (23). Shokouhinejad et al. (24) evaluated the effect of acidic environment on pushout bond strength of MTA cement and concluded that MTA treated with PBS had significantly higher bond strength than the group treated with butyric acid; this shows that the $\mathrm{pH}$ of 7 is an ideal $\mathrm{pH}$ for the setting reaction of MTA to occur. Thus, placement of a cotton pellet dipped in saline on the MTA during its setting increa- ses its bond strength. In the current study, saline-treated group ranked second after the control group in terms of the highest push-out bond strength.

Jantarat et al. (7) assessed the efficacy of the new formulation of EDTA (Smear Clear) for elimination of smear layer from the root canal dentin and showed that Smear Clear eliminated the smear layer from all coronal, middle and apical surfaces of the root but $\mathrm{NaOCl}$ was not capable of eliminating the smear layer from root canal surfaces. They concluded that Smear Clear had the highest efficacy for smear layer removal and cleaning of the root canal walls. Venghat et al., in their in vitro study (25) revealed that Smear Clear was less efficient for smear layer removal than EDTA. Da Silva et al. (26) evaluated the efficacy of Smear Clear and EDTA for elimination of smear layer of permanent teeth after root canal instrumentation and concluded that Smear Clear had an efficacy similar to that of $14.3 \%$ EDTA for smear layer removal from the root canals, and the differences with the control group were statistically significant. To the best of our knowledge, no previous study has evaluated the effect of Smear Clear on push-out bond strength of MTA to dentin. In the current study, MTA treated with Smear Clear had significantly lower bond strength than the MTA treated with saline and the control group $(P<0.05)$. Further studies are required to assess the effect of Smear Clear on hydration of MTA. Scanning electron microscopic analyses are also required to assess MTA cement after exposure to Smear Clear.

Mode of failure in all MTA groups in our study was evaluated under a stereomicroscope at $\times 40$ magnification. Mode of failure was mixed in most samples (Table 2), which was in contrast to the findings of Guneser et al., (21) and Sobhnamayan et al. (6). This difference is due to the fact that in the study by Sobhnamayan et al., (6) calcium enriched mixture cement was used, which is different from MTA in terms of the size of particles (22). In the study by Guneser et al., the powder/water ratio of MTA cement was 3:1, which was different from the powder/water ratio of MTA cement in the current study. In the study by Cecchin et al., (19) mode of failure was mainly mixed in groups treated with CHX and saline (control), which was in line with our results.

\section{Conclusions}

In the current study, the push-out bond strength of the MTA group exposed to saline was the highest. Immersion in Smear Clear, $\mathrm{CHX}$ and $\mathrm{NaOCl}$ irrigants decreased the bond strength of MTA to dentin following the first 10 minutes of its setting.

\section{References}

1. Hong ST, Bae KS, Baek SH, Kum KY, Shon WJ, Lee W. Effects of root canal irrigants on the push-out strength and hydration behavior of accelerated mineral trioxide aggregate in its early setting phase. Journal of endodontics. 2010;36:1995-9. 
2. Çelik D, Er K, Serper A, Taşdemir T, Ceyhanlı KT. Push-out bond strength of three calcium silicate cements to root canal dentine after two different irrigation regimes. Clinical oral investigations. 2014;18:1141-6.

3. Smith JB, Loushine RJ, Weller RN, Rueggeberg FA, Whitford GM, Pashley DH, et al. Metrologic evaluation of the surface of white MTA after the use of two endodontic irrigants. Journal of endodontics. 2007;33:463-7.

4. Formosa L, Mallia B, Camilleri J. Push-out bond strength of MTA with antiwashout gel or resins. International endodontic journal. 2014;47:454-62.

5. Sluyk S, Moon P, Hartwell G. Evaluation of setting properties and retention characteristics of mineral trioxide aggregate when used as a furcation perforation repair material. Journal of Endodontics. 1998;24:768-71.

6. Sobhnamayan F, Adl A, Shojaee NS, Gavahian S. The Effect of Chlorhexidine on the Push-Out Bond Strength of Calcium-Enriched Mixture Cement. Iranian endodontic journal. 2015;10:59.

7. Jantarat J, Yanpiset K, Harnirattisai C. Evaluation of smear layer removal by a new EDTA formula on root canal dentin: a scanning electron microscopic study. Submitted for publication. 2004.

8. Dunavant TR, Regan JD, Glickman GN, Solomon ES, Honeyman AL. Comparative evaluation of endodontic irrigants against Enterococcus faecalis biofilms. Journal of Endodontics. 2006;32:527-31.

9. Yan P, Peng B, Fan B, Fan M, Bian Z. The effects of sodium hypochlorite (5.25\%), Chlorhexidine (2\%), and Glyde File Prep on the bond strength of MTA-dentin. Journal of Endodontics. 2006;32:58-60.

10. Loxley EC, Liewehr FR, Buxton T, McPherson J. The effect of various intracanal oxidizing agents on the push-out strength of various perforation repair materials. Oral Surg Oral Med Oral Pathol Oral Radiol Endod.2003;95:490-4.

11. Collares F, Portella F, Rodrigues S, Celeste R, Leitune V, Samuel S. The influence of methodological variables on the push-out resistance to dislodgement of root filling materials: a meta-regression analysis. International endodontic journal. 2015 Sep 1. [Epub ahead of print] 12. Pane ES, Palamara JE, Messer HH. Critical evaluation of the push-out test for root canal filling materials. Journal of endodontics. 2013;39:669-73.

13. Camilleri J, Montesin FE, Brady K, Sweeney R, Curtis RV, Ford TRP. The constitution of mineral trioxide aggregate. Dental Materials. 2005;21:297-303.

14. Pascon FM, Kantovitz KR, Sacramento PA, Nobre-dos-Santos M, Puppin-Rontani RM. Effect of sodium hypochlorite on dentine mechanical properties. A review. Journal of dentistry. 2009;37(12):903-8.

15. Bryce G, O’Donnell D, Ready D, Ng YL, Pratten J, Gulabivala K. Contemporary root canal irrigants are able to disrupt and eradicate single-and dual-species biofilms. Journal of Endodontics. 2009;35:1243-8.

16. Nagas E, Cehreli Z, Uyanik M, Vallittu P, Lassila L. Effect of several intracanal medicaments on the push-out bond strength of ProRoot MTA and Biodentine. International endodontic journal. 2016;49:184-8.

17. Uyanik MO, Nagas E, Sahin C, Dagli F, Cehreli ZC. Effects of different irrigation regimens on the sealing properties of repaired furcal perforations. Oral Surg Oral Med Oral Pathol Oral Radiol Endod. 2009;107:e91-e5.

18. Basrani BR, Manek S, Sodhi RN, Fillery E, Manzur A. Interaction between sodium hypochlorite and chlorhexidine gluconate. Journal of endodontics. 2007;33:966-9.

19. Cecchin D, de Almeida JFA, Gomes BP, Zaia AA, Ferraz CCR. Effect of chlorhexidine and ethanol on the durability of the adhesion of the fiber post relined with resin composite to the root canal. Journal of endodontics. 2011;37:678-83.

20. Agrawal A, Paul B, Mantri S, Dube K. The Effect of Different Irrigation Regiments on the Push out Bond Strength of MTA Fillapex Sealer to Dentin. British Journal of Medicine and Medical Research. $2015 ; 9$.

21. Guneser MB, Akbulut MB, Eldeniz AU. Effect of various endodontic irrigants on the push-out bond strength of biodentine and con- ventional root perforation repair materials. Journal of endodontics. 2013;39:380-4.

22. Reyes-Carmona JF, Felippe MS, Felippe WT. The biomineralization ability of mineral trioxide aggregate and Portland cement on dentin enhances the push-out strength. Journal of endodontics. 2010;36:286-91.

23. Al-Zubaidi AK, Al-Azzawi AKJ. The effect of various endodontic irrigants on the sealing ability of Biodentine and other root perforation repair materials (In vitro study). Journal of Baghdad College of Dentistry. 2014;26:1-8

24. Shokouhinejad N, Nekoofar MH, Iravani A, Kharrazifard MJ, Dummer PM. Effect of acidic environment on the push-out bond strength of mineral trioxide aggregate. Journal of Endodontics. 2010;36:871-4.

25. Venghat S, Hegde MN. Comparative Evaluation of Smear Layer Removal Efficacy Using QMix 2in1, Chitosan, Smear Clear and Glyde. British Journal of Medicine and Medical Research. 2016;13:1-8

26. da Silva LAB, Sanguino ACM, Rocha CT, Leonardo MR, Silva RAB. Scanning electron microscopic preliminary study of the efficacy of SmearClear and EDTA for smear layer removal after root canal instrumentation in permanent teeth. Journal of endodontics. 2008;34:1541-4

\section{Acknowledgments}

This research has been supported by Tehran University of Medical Sciences, International Campus.

\section{Conflict of Interest}

The authors deny any conflicts of interest. 